\title{
The effects of a nurse-led supportive care delivery including Complementary and Integrative Medicine (CIM) on breast and gynaecologic cancer patients' quality of life
}

Klafke, Nadja ${ }^{1}$; Mahler, Corneliaㅁ; Uhlmann, Lorenz²; von Hagens, Cornelia3; Bentner, Martina ${ }^{1}$; Schneeweiss, Andreas 4 ; Müller, Andreas ${ }^{5}$; Szecsenyi, Joachim ${ }^{1}$; Joos, Stefanie ${ }^{6}$

\section{Introduction \& Objectives}

Although conventional oncological treatment has led to improvement of survival rates and better treatment outcomes, chemotherapy (CHT) treatment is still associated with impairment on patient-reported outcomes (PROs) and subsequent detriment on Quality of Life (QoL) [1]. Early and regular symptom management is therefore crucial for patients undergoing CHT. Many CIMs have the potential of improving symptoms [2], however, an integrated nurse-led supportive care program including CIM counselling and applications has not yet been evaluated. The aim of the CONGO (COmplementary Nursing in Gynecologic Oncology)-study was to investigate, in two different healthcare settings, the effects of an implemented CIM supportive therapy intervention on QoL, primarily, and associated PROs, secondary, in patients starting a new regimen of $\mathrm{CHT}$ [3].

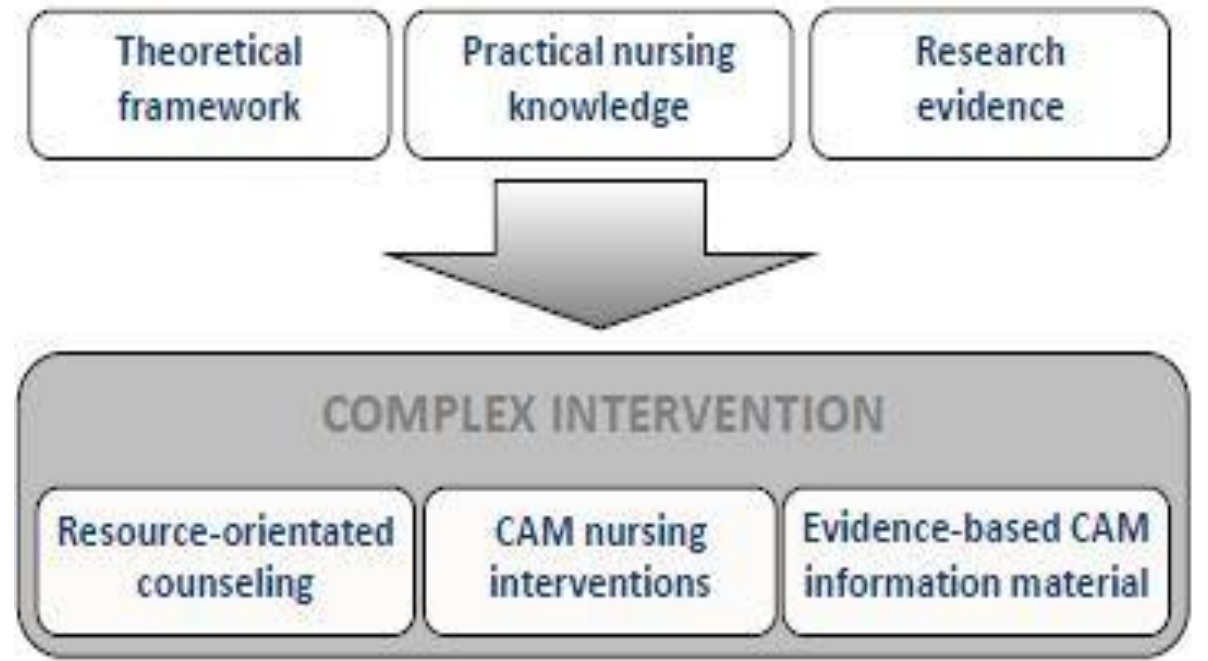

\section{Methods}

A randomized controlled study design was applied to recruit female cancer patients in a University hospital (National Center of Tumor Diseases, NCT, Heidelberg) and a community hospital (Städtisches Klinikum Karlsruhe, SKK). Patients in the intervention group (IG) received routine care plus the CIM intervention based upon their needs and preferences at each new CHT cycle. Routine care for patients in the control group (CG) was not changed. Trained oncology nurses were responsible for conducting the intervention consisting of three complementing components [4] (see Figure 1): 1) resource-oriented counselling, 2) CIM nursing interventions for symptom management, 3) evidence-based information material on CIM (see Figure 1). Patients in the IG received these CIM interventions at each new $\mathrm{CHT}$ cycle as well as with instructions for home use.

QoL was measured with the EORTC-QLQ-C30 at four time points: T1 (baseline, prior to CHT), T2 (midline of CHT), T3 (end of CHT, max. 24 weeks), and follow-up T4 (6 months after completion of $\mathrm{CHT}$ ). Mixed linear models with fixed and random factors were applied to test for group effects.

\section{Results}

From $7 / 2014$ to 2/2016, 251 patients were randomized into the study ( $87 \%$ response rate). The sample (median 51 years) was balanced regarding cancer type ( $84 \%$ breast cancer), stage of cancer $(86 \%$ curative treatment), and form of treatment $(50 \%$ neoadjuvant).

No group effects on global QoL were analyzed at T3 (estimate $-1.04 \quad[-4.89 ; 2.809], p=0.596)$, but at follow-up T4 (estimate $6.643[1.65 ; 11.64](p=0.010)$ (see Figure 2). At T4, IG patients did also experience significant better emotional functioning (effect estimate: 8.196 [2.328; 14.07], $p=0.007)$ and less fatigue (effect estimate: -7.04 [-13.2;-.840], $p=0.027$ ).

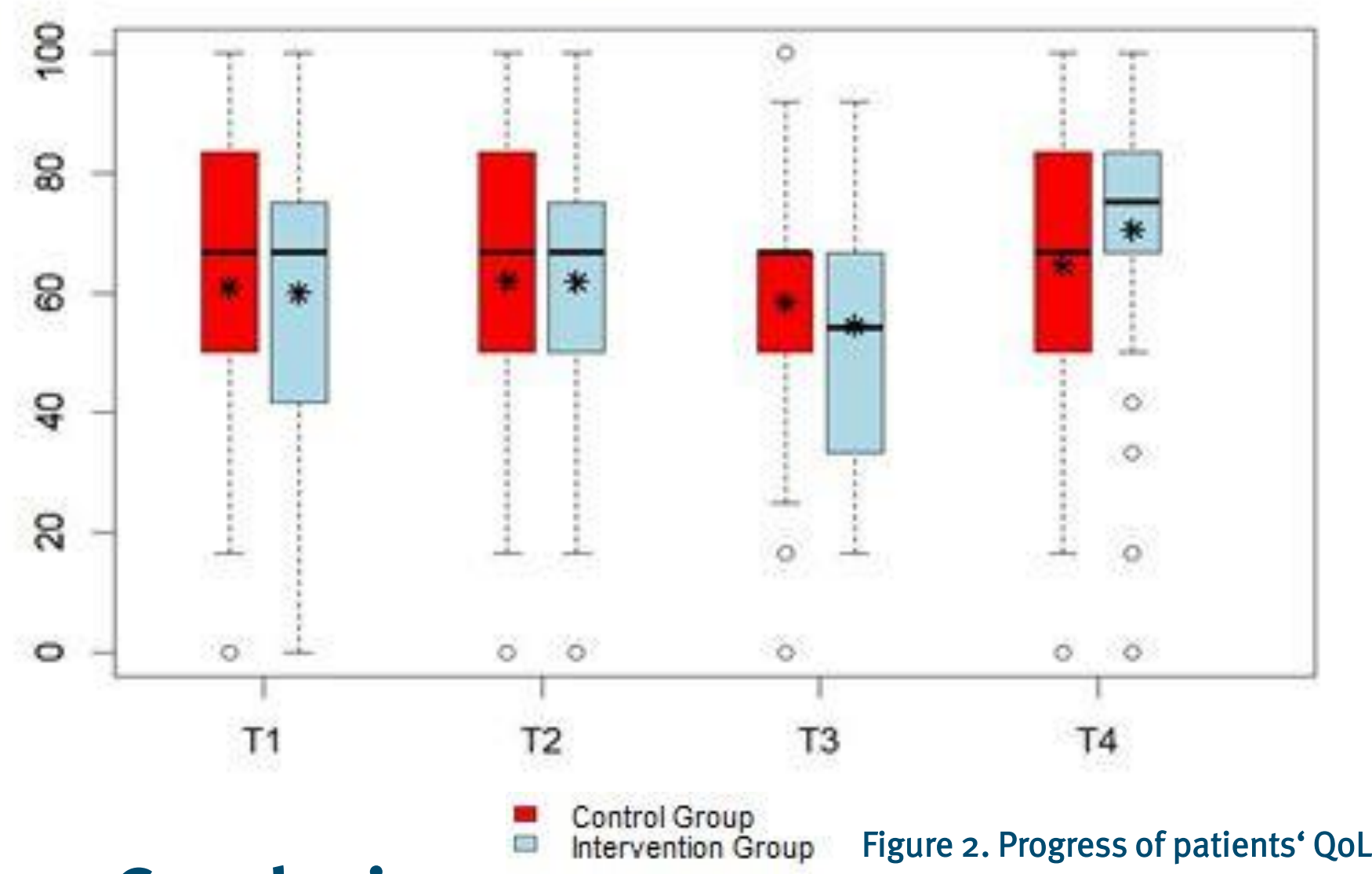

\section{Conclusion}

The findings of the CONGO-study indicate that nursing symptom management with CIM, administered regularly during chemotherapy, has the potential to improve patients' quality of life experience. Based on the assumption that the delayed effect is due to a strenghtening of patients ' self-management and coping strategies, integrating nurse-led CIM interventions might be an effective approach to individually address patients' needs. 